\title{
Maternal Health Care in Cox's Bazar, Bangladesh: A Survey of Midwifery Experience at Hope Foundation and a Review of the Literature
}

\author{
Iftikher Mahmood1, Hannah Bergbower'1, Arman Mahmood1', Annekathryn Goodman²* \\ ${ }^{1}$ Hope Foundation for Women and Children of Bangladesh, Cox's Bazar, Bangladesh \\ ${ }^{2}$ Department of Obstetrics and Gynecology, Massachusetts General Hospital, Boston, MA, USA \\ Email: iumahmood@gmail.com, hbergbower@gmail.com, arman.mahmood12@gmail.com, *agoodman@mgh.harvard.edu
}

How to cite this paper: Mahmood, I., Bergbower, H., Mahmood, A. and Goodman, A. (2019) Maternal Health Care in Cox's Bazar, Bangladesh: A Survey of Midwifery Experience at Hope Foundation and a Review of the Literature. Open Journal of Obstetrics and Gynecology, 9, 1624-1637. https://doi.org/10.4236/ojog.2019.912158

Received: December 2, 2019

Accepted: December 28, 2019

Published: December 31, 2019

Copyright $\odot 2019$ by author(s) and Scientific Research Publishing Inc. This work is licensed under the Creative Commons Attribution International License (CC BY 4.0).

http://creativecommons.org/licenses/by/4.0/

(c) (i) Open Access

\begin{abstract}
Background: The Ministry of Health and Family Welfare of the Government of Bangladesh developed a midwifery education strategy in 2011 and starting in 2013, BRAC University developed a three-year Diploma in Midwifery. A survey was developed to understand the challenges of the newly graduated midwives and to identify resource and educational needs. The survey feedback will help develop and strengthen curriculum for primary and postgraduate midwifery training. Methods: A 30-question survey was given to midwives at the Hope Hospital in Cox's Bazar and at the birth centers in the surrounding rural communities. Questions explored the midwives' clinical experience, patient problems in the prenatal, intrapartum, and postnatal period, and asked about what education; training and clinical resources were needed. Results: Thirty-two midwives answered the surveys. The midwives' average time from graduation from the midwifery diploma program was sixteen months. All the respondents felt comfortable managing most maternal issues but felt further training was important. They also identified the lack of many clinical resources including blood products, medicines, vaccines, and ultrasound. They identified significant maternal health issues among their patients including adolescent pregnancy, malnutrition, anemia, sexual violence, pregnancy-induced hypertension, hemorrhage, low birth weight infants, prolonged and obstructed labors. Conclusion: Training and increasing the number of midwives are crucial strategies for reducing maternal and neonatal mortality. There is a significant need for long-term placement and commitment of midwives to rural areas in Bangladesh. Health systems and organizations employing midwives must be accountable for the continuing education,
\end{abstract}


mentorship, and supportive needs of midwives.

\section{Keywords}

Bangladesh, Maternal Mortality, Maternal Healthcare, Midwives, Midwifery, Midwifery Training, Cox’s Bazar, Adolescent Pregnancy, Pregnancy Outcomes, Medical Decision Making, Sexual Reproductive Healthcare, Hope Foundation

\section{Introduction}

Bangladesh, a country of 170 million people, has an annual birth rate of 3.1 million [1]. Only $47 \%$ of births occur in a health facility. Since 2000, Bangladesh has accomplished a 50 percent decrease in maternal mortality [2]. With a current maternal mortality rate of 196 maternal deaths per 100,000 live births, there is a need to re-examine and change the current infrastructure for women's reproductive healthcare [3].

There are many barriers to safe maternal health care. Barriers include a lack of medical resources such as infrastructure, shortages of medicines, and limited training of healthcare providers [4]. Challenges to staff training include a limited knowledge of and experience with labor management, lack of prenatal screening of patients, the need for evidence-based protocols and appropriate indications for cesarean delivery, and lack of quality improvement (QI) support [5] [6]. In addition, patient factors such as lack of education, lack of autonomy in medical decision-making, and the financial burden of medical care to the family increase the likelihood of poor obstetrical outcomes [7].

The United Nations Population Fund (UNFPA) supports the midwifery profession, as improved access to obstetrical care is the most effective solution to poor birth outcomes [8]. The widespread integration of midwifery in Bangladesh has been a key priority over the last decade. It is projected that, in order to meet the national need, an estimated 22,000 midwives must be trained and deployed [9]. The Ministry of Health and Family Welfare (MoHFW) of the Government of Bangladesh developed a plan for midwifery education strategy in 2011 [10]. Professional midwives were introduced into Bangladesh in 2013 and in January 2013, BRAC University (BRACU) developed a three-year Diploma in Midwifery, the Developing Midwives Project [11]. The first class of diploma-licensed midwives graduated in 2016 [12]. However, there remains a significant need for and shortage of midwives in Bangladesh.

Improvement in women's reproductive health in Cox's Bazar, a district of the division of Chittagong in the Southern region of Bangladesh, lags behind the rest of the country [13]. Bangladesh's family planning goal for Cox's Bazar is for fifty percent of health clinics to deliver postpartum family planning services by 2020 [14]. A recent needs assessment identified a high rate of home deliveries; $68 \%$ in the Teknaf Upazila (subdistrict) and 53\% in the Ukhia Upazila [15]. A new chal- 
lenge to adequate reproductive healthcare services in the Cox's Bazar district has emerged since the exodus of Rohingya refugees from Myanmar in 2017. Within the Rohingya population of Cox's Bazar, there is a crucial need for sexual and reproductive healthcare for the estimated 300,000 women and girls of reproductive age, of which ten percent are pregnant [16].

One Bangladeshi non-government organization, Hope Foundation for Women and Children of Bangladesh, has worked in the Cox's Bazar district since 1999 and has been an instrumental partner in the creation and deployment of the midwifery workforce in Bangladesh [17]. Beginning as a one-room clinic in 1999, the foundation opened Hope Hospital, a 40-bed maternity and fistula hospital, in 2005 that is strategically located in the rural area of Ramu, Cox's Bazar. Hope Hospital is now a referral center for complex obstetrical and gynecological patients. The mission of the organization is to serve those with the greatest need and provide access to care in rural, underserved areas. In addition, Hope Foundation has developed seven midwife-run birthing centers in the rural areas of the district. The staff of Hope Foundation has been delivering maternity care to Rohingya refugees since 2017 and opened Hope Field Hospital for Women in the refugee camp, as well as eight sexual and reproductive health $(\mathrm{SRH})$ centers, in high-need areas, within different camps.

In 2013, Hope Foundation opened a three-year midwifery diploma program with support from BRAC University. To date, there have been three graduating classes of seventy-eight students. Fifty more students are currently enrolled in the program, with the intake of the seventh batch in progress.

Our goal was to survey the graduating midwives from the Hope Foundation program who are working in Cox's Bazar. The survey information adds to the existing literature, helps to understand the current challenges of midwives in this community, and will help update the curriculum to meet midwifery training needs.

\section{Methods}

With hospital ethics committee approval, a 30-question survey was developed to evaluate the experience of the midwives who had graduated from the Hope Foundation diploma program. The faculty of the program created the questionnaire to assess the challenges, resource limitations, and patient factors that were identified by the midwives. The questionnaire was given to midwives at the Hope Hospital in Cox's Bazar and at the birth centers in the surrounding rural communities. All midwives who had graduated from the Hope Foundation program were included in the survey. Paramedics who also deliver babies at the birth centers and traditional birth attendants were excluded from the survey. Table 1 summarizes the questions.

\section{Results}

Thirty-two midwives answered the survey. Table 2 summarizes their sociodemographic characteristics. The average age of the midwives was 22 years. All the 
Table 1. Questionaires for midwives.

\section{A. Midwife Experience}

1) How many years have you been a midwife?

2) How many women do you deliver per month?

3) What additional training do you want?

4) How should additional training be given?

5) What additional resources do they need in the hospital or birthing center?

\section{B. Patient Care Problems: Pregnant Patients and during Labor}

Prenatal Care:

1) What are the most serious health problems?

2) Is it difficult for patients to come for appointments?

3) Do patients have trouble getting transportation to come to the birthing center or hospital?

4) Do patients understand the importance of good prenatal care on birth outcomes?

5) What percentage of women receive prenatal care?

6) Who makes medical decisions for the patient's healthcare?

7) Do the patients get enough to eat at home?

8) Is there a problem with teenage pregnancy?

Labor and Delivery

1) Do you feel comfortable managing bleeding after delivery?

2) What are the most serious problems during labor?

3) Are you comfortable repairing a vaginal laceration?

5) How comfortable are you when working with non-midwife colleagues?

6) What percentage of patients are referred from the birthing center to the hospital?

\section{Postpartum}

1) What are the most difficult postpartum problems?

2) Do you have an opportunity to teach about breast-feeding?

3) Do you have an opportunity to counsel and prescribe birth control?

4) What percent of mothers breastfeed their newborns?

5) What percent of women use family planning?

Table 2. Sociodemographic characteristics of midwives.

\begin{tabular}{cc}
\hline Characteristic $\mathbf{n}=32$ & Range (mean) \\
\hline Age & $20-24$ years (22 years) \\
Time since graduation \\
from midwifery program \\
$\begin{array}{c}\text { Percent of midwives from local } \\
\text { Cox's Bazar communities }\end{array}$ \\
Location of midwife practice \\
\end{tabular}


midwives were recruited from the Cox's Bazar region. Eleven worked in the Hope Field Hospital in the Rohingya refugee camp. Five practiced at Hope Hospital and sixteen worked in birthing centers in the remote rural areas. The midwives' personal practice experience ranged from two months to 21 months, with an average time from graduation from the midwifery diploma program of sixteen months. All midwives answered that they were comfortable working with non-midwife colleagues. All respondents said they were comfortable managing bleeding and vaginal lacerations but wanted further training or mentoring. All the respondents felt further training was important and the majority (90\%) thought that monthly training workshops would be helpful. Table 3 lists the topics that midwives felt would be important for continued education.

When asked about resources needed, all respondents identified the following scarce resources: blood products, medicines, vaccinations and more supplies. All midwives also identified the need for ultrasound and an expert ultrasound practitioner. One midwife responded, "Everything and we need a doctor".

Overall deliveries per month ranged from five to fifty. The average number of births per month was twelve at the Field Hospital, twenty at Hope Hospital, and eleven at each birthing center. Table 4 describes the prenatal problems the midwives identified that the patients experienced. The midwives reported 50 to 90 percent of patients received prenatal care at all sites. The midwives felt that most patients understood the importance of prenatal care but many required counseling and education.

Thirty percent of respondents said that their patients had trouble getting to the health centers. The reasons given included lack of finances, lack of transportation, difficulties with the husband, and difficulties with the in-laws. One respondent reported that her patients have trouble getting care because of religious reasons. When asked about who made the medical decisions about obstetrical care, $100 \%$ of respondents identified the husband as the primary decision maker

Table 3. Topics for continuing midwifery education.

Pelvic examination
Cervical cancer screening
Sexually transmitted infections
Suturing
Laceration repair
Difficult deliveries
Management of breech delivery
Management of intrapartum and postpartum hemorrhage
Family planning
Oral contraception
Menstrual regulation


and $78 \%$ reported both husband and parents or in-laws as the decision makers. Sixty-three percent of respondents said that their patients did not get enough to eat and $81 \%$ felt that adolescent pregnancies were a problem. Identified problems with adolescent pregnancies included anemia, malnutrition, preeclampsia, cephalo-pelvic disproportion, low birth weight babies, and increased fetal demise.

Table 5 lists both intrapartum and postpartum problems identified by the midwives. Referrals from the birthing centers and Field hospital to Hope Hospital in Ramu or the government hospital occurred with an average of $20 \%$ of the deliveries. Seventy-percent of deliveries at Hope Hospital were referred in from another location. All respondents felt that they had a good system for referring complicated patients, including access to an ambulance.

All the respondents discussed and taught breast-feeding and family planning. One midwife commented that while she taught about breast-feeding, there was no private space in the health center for her patients to breast-feed. Seventy-five

Table 4. Prenatal care: identified diagnoses and concerns.

Hypertension
Diabetes
Preeclampsia
Anemia
Malnutrition
Bleeding
Preterm labor
Gastritis
Back pain
Lower abdominal pain
Sexual violence
Adolescent pregnancy

Table 5. Intrapartum and postpartum complications of pregnancy.

\begin{tabular}{cc}
\hline Intrapartum & Postpartum \\
\hline Preeclampsia and eclampsia & Postpartum hemorrhage \\
Prolonged labor & Hypovolemic shock \\
Obstructed labor & Eclampsia, Convulsions \\
Breech presentation & Infection \\
Shoulder dystocia & Septic shock \\
Retained placenta & Depression \\
Hemorrhage & \\
Birth Asphyxia & \\
\hline
\end{tabular}


to $100 \%$ of patients breastfed their newborn babies. Fifty-five percent of postpartum patients used family planning methods (ranged from $20 \%$ to $80 \%$ ).

\section{Discussion}

Our survey of thirty-two young midwives in the Cox's Bazar district of Bangladesh gives a preliminary picture of the successes and challenges experienced by midwives in a rural and resource-limited region. The midwives successfully increased maternal healthcare to over forty women each month. The respondents of this survey identified important potentially life-threatening obstetrical issues in prenatal, intrapartum, and postpartum periods that must be addressed in the initial curriculum of the midwife diploma program and in ongoing continuing midwifery education. The survey reinforced the need for mentoring and continued training but also documented that the graduating midwives feel competent to perform their duties in fairly austere environments. Additionally, Hope Foundation has set-up an efficient referral pathway for problem pregnancies with a local ambulance service and the receiving district hospitals. The midwives also identified the need and scarcity of important resources such as blood products, medicines, vaccines, and ultrasound expertize that is essential to improve maternal outcomes. The midwife respondents also identified significant challenges for their patient population including adolescent pregnancies, patient lack of autonomy to make their own medical decisions, malnutrition, and potential sexual violence. These important findings support the importance of understanding the social determinants of maternal health in Bangladesh. Our findings are consistent with other studies, which are reviewed here.

Barriers to Sexual and Reproductive Health Care

In Bangladesh, there is both a shortage of doctors and a geographic mal-distribution of physicians in the country. Approximately 3.05 doctors and 1.07 nurses per 10,000 population lead to a vacancy of $36 \%$ of healthcare worker positions [18]. This severe shortage of medical professionals reduces available resources in sexual and reproductive healthcare. In a survey of two district and twelve sub-district hospitals in Thakurgaon and Jamalpur in Bangladesh, hospital staff identified several key factors that affected the quality of obstetrical care: shortage of staff and logistics; lack of laboratory support; underuse of patient-management protocols; a lack of training; and insufficient supervision [6]. Doctors were unable to provide optimal care because of the high volume of patients. In the Cox's Bazar area, a lack of human resources and hospital bed capacity leads to gaps in basic emergency obstetrical care [19]. One solution to the lack of staffing is the training of midwives and addition of midwifery expertise for improved access to obstetrical care. While new Bangladeshi midwives have been coming into the healthcare workforce since 2016, most midwives in remote areas are young and inexperienced with little access to mentoring and back up [19].

In addition to these barriers that healthcare providers face, rural patients en- 
counter significant barriers in accessing those providers. While nearly $70 \%$ of the population in Bangladesh lives in rural areas, less than $20 \%$ of healthcare workers provide medical care in these areas [20]. This leaves patients turning to untrained providers and a substantial burden being placed on providers practicing in these areas. The Hope midwifery training program has employed a model of rural recruitment so that, after training, midwives return to their home communities as cultural brokers and maternal health leaders.

An additional concern was the lack of utilization of medical protocols to provide standardized care by physicians, nurses, and other healthcare professionals [6]. There was a lack of continued obstetrical refresher training and supervision for healthcare professions that was thought to reduce quality of care [21].

Another barrier to good obstetrical care is the inability of pregnant women to control their own medical care. Two-thirds of the world's women are illiterate and sixty percent of the world's poor are girls and women [22]. In Bangladesh, husbands and in-laws guide medical decision-making. Our survey confirmed that none of the patients had a voice in their own obstetrical care. In a survey of 8753 women in Bangladesh, the risk factors for not accessing maternal healthcare included no formal education, of Islamic faith, from poorest wealth quintile, living in rural areas and with low autonomy [23]. Another survey documenting trends and inequities of maternal care in Bangladesh identified maternal education as the most powerful indicator of healthcare access [24].

Socio-economics guide medical decision-making. There is a direct correlation in Bangladesh between socio-economic status and maternal mortality [25]. Additionally, gender inequality plays a significant role in Bangladesh in women's access to maternal health care [26] [27].

Mode of Deliveries and Outcomes

In Bangladesh, there is a lack of universal data collection on birth outcomes. In rural areas, family members and friends provided over seventy five percent of birth and postpartum care at home and only twenty percent of pregnant women had access to medically attended care [28]. A survey of over 100,000 women in rural and urban settings revealed that rural women experience complications of pregnancy and postpartum period in over half of deliveries including pre-eclampsia, infections, and urinary incontinence [29]. Another study of postpartum Bangladeshi women identified hemorrhage, eclampsia, pain, and prolonged labor as significant problems [30]. Maternal deaths, $80 \%$ of which occur in the postpartum period, are secondary to hemorrhage, pre-eclampsia, infection, and septic abortion [31].

Selected studies suggest that the overall cesarean section rate is higher than global recommendations. A retrospective survey of an area of Dhaka, Bangladesh identified a $35 \%$ cesarean delivery rate with only $1.4 \%$ conducted due to absolute maternal indications [32]. According to data produced by Save the Children (2019) the cesarean section rate in private facilities is approximately $80 \%$ and has increased by $51 \%$ over the past two years [33]. 
Midwives have been described as a key solution to reducing cesarean rates globally, including in Bangladesh [34]. Trained midwives have the skills and knowledge of normal labor patterns and safe birth and postpartum management that enable them to differentiate between the variations and identify abnormal signs that require intervention. Not only is a reduction in cesarean rates crucial in improving maternal outcomes, but midwives' impact on reducing surgical intervention in birth has the potential to yield substantially positive economic outcomes on investment as well [8].

Midwifery

Midwives must be equipped with the knowledge, skills, and resources to effectively provide care in accordance with international standards. The ability of midwives to impact maternal health outcomes will depend on the quality of education, definition of scope of practice, support and supervision, appropriate back-up and teamwork. One educational challenge is retaining teachers in midwifery diploma programs. When BRACU recruited qualified faculty to remote and underserved areas, 23 out of 30 faculty (both doctors and nurse-midwives) left the program [11]. Retention strategies have included financial incentives and focusing on nurse-midwife faculty. Another strategy has been to recruit young women from rural communities into the midwifery-training program so that they will return home and create sustainable maternity care access in their communities.

In addition, midwives, as is true for physicians, must have adequate supplies and resources and have access to timely transfer and referral for complicated cases [35]. One study analyzed the tensions and conflicts present in developing complex health systems in Bangladesh using the tool called Complex Adaptive System (CAS) [36]. The common goal of all professionals interviewed (government officials, medical leaders, clinicians) was to save lives. The recommendations focused on the importance of collaboration between policy makers and medical leadership to develop the infrastructure for a midwifery profession. This requires tight communication and shared educational competencies and goals.

There are three layers of barriers to care that midwives have to navigate in Bangladesh: social, professional, and economic [37]. These barriers were first described as barriers that are intrinsically enmeshed in a society and impact the quality of childbirth and the ability for midwives to deliver good care [38]. Midwives need to be included in professional societies that focus on SRH [39]. Social barriers describe the lack of resources targeted for women's healthcare as a result of underlying gender inequality in a particular culture. Economic barriers pertain to underpaying midwives for their work and how low payment relates to lack of society's value for the work of a midwife. Professional barriers describe both the lower status of midwives in the health community and the lack of infrastructure resources given to these professionals such as supplies, medicines, and health facility space.

To further elaborate on these barriers, midwifery in Bangladesh is a female-led 
profession, creating challenges that must be socially, professionally, and economically navigated and that have substantial impact on opportunity and advancement. The story of a woman entering this profession itself is of monumental importance considering the challenges at play. In addition, continuing education and mentorship opportunities, as well as lack of professional society organization and involvement, create barriers for midwives as they attempt to develop. Continuing educational curriculum, necessary to maintain skills, may not be available for midwives who practice in remote and resource-limited environments.

To understand how to create an infrastructure for the midwifery profession in Bangladesh, thirty-three government officials, policy makers, and donors were interviewed [40]. Collaboration and political commitment are mandatory. Only when there is buy-in by policy makers and medical professionals and agreement on guidelines, will midwives be included as part of the national health workforce.

Our survey of thirty-two midwives supports the findings that there is an important need for midwifery care in Bangladesh. The survey is the first step in analyzing the experience of the newly formed midwifery diploma program at Hope Foundation. The limitations of our survey are that we do not know the incidence and prevalence of the various pregnancy-related conditions that the midwives identified. In addition, we do not have data on maternal and neonatal outcomes from this survey. Finally, the survey did not capture the varied and individual experience of midwives working alone in a resource-limited environment who are facing complex pregnancy-related problems. The next step is to construct a survey of midwives that uses qualitative interviews. This will allow a deeper understanding of the conditions within which midwives work and help gain greater insight into the issues that pregnant women struggle with. Future studies must include careful documentation of diagnoses and short and long-term outcomes. This data is crucial for quality assurance and identification of sentinel events that can lead to changes in policies, medical protocols, and ongoing clinician training [41].

\section{Conclusion}

Our survey identified both sociodemographic factors of the patients such as adolescent pregnancy, malnutrition, and lack of medical decision making capacity and lack of clinical resources as major challenges for midwives working in the field. Improvement of maternal care can come from a bigger presence of midwives in sexual and reproductive healthcare. However, our midwives have identified the need for more training in complex obstetrical care, social service support, and the need to increase medication and equipment on site at the birthing centers. Wherever midwives may be located or practicing in Bangladesh, support, mentorship and regular training updates must be provided. There is a significant need for long-term placement and commitment of providers to rural 
areas, with the sustainable solution being oriented towards training individuals from these communities who will desire to return home. Recruitment of young women living in rural, underserved areas, into the midwifery profession not only supports sustainable solutions for maternal health in these communities, but also provides opportunities for education and economic empowerment. Health systems and organizations employing midwives must be accountable to the continuing education, mentorship, and supportive needs of midwives in ensuring they are equipped with the training and resources necessary to successfully navigate the deep challenges they are facing. There is a substantial unmet need in terms of professional society representation, to stand with midwives and ensure their needs are met. Midwives are crucial in reducing maternal and neonatal mortality, reducing unnecessary cesarean rates, increasing normal vaginal delivery rates, and creating a standard expectation of continuity of care through the implementation of the Golden Chain model of care.

\section{Conflicts of Interest}

The authors have no conflicts of interest. Dr. Iftikher Mahmood is the CEO of Hope Foundation, which is a non-profit organization.

\section{References}

[1] Mitra and Associate (2014) BDHS Bangladesh Demographic Health Survey. Dhaka. https://dhsprogram.com/pubs/pdf/FR311/FR311.pdf

[2] WHO World Health Organization (2015) Maternal Mortality in 1990-2015, Bangladesh. Maternal Mortality Estimation Inter-Agency Group. https://www.who.int/gho/maternal_health/countries/bgd.pdf

[3] National Institute of Population Research and Training (NIPORT) (2016) BMMS Bangladesh Maternal Mortality and Health Care Survey. https://www.measureevaluation.org/resources/publications/tr-17-218

[4] Wichaidit, W., Alam, M., Halder, A.K., Unicomb, L., Hamer, D.H. and Ram, P.K. (2016) Availability and Quality of Emergency Obstetric and Newborn Care in Bangladesh. The American Journal of Tropical Medicine and Hygiene, 95, 298-306. https://doi.org/10.4269/ajtmh.15-0350

[5] Chowdhury, S., Hossain, S.A. and Halim, A. (2009) Assessment of Quality of Care in Maternal and Newborn Health Services Available in Public Health Care Facilities in Bangladesh. Bangladesh Medical Research Council Bulletin, 35, 53-56. https://doi.org/10.3329/bmrcb.v35i2.3044

[6] Islam, F., Rahman, A., Halim, A., Eriksson, C., Rahman, F. and Dalal, K. (2015) Perceptions of Health Care Providers and Patients on Quality of Care in Maternal and Neonatal Health in Fourteen Bangladesh Government Healthcare Facilities: A Mixed-Method Study. BMC Health Services Research, 15, 237.

https://doi.org/10.1186/s12913-015-0918-9

[7] Islam, M.R., Rahman, M.S., Islam, Z., Nurs, C.Z., Sultana, P. and Rahman, M.M. (2017) Inequalities in Financial Risk Protection in Bangladesh: An Assessment of Universal Health Coverage. International Journal for Equity in Health, 16, 59. https://doi.org/10.1186/s12939-017-0556-4

[8] UNFPA United Nations Population Fund (2014) The State of the World's Midwi- 
fery 2014. A Universal Pathway. A Woman's Right to Health. New York. https://www.unfpa.org/sowmy

[9] UNFPA United Nations Population Fund (2018) Bangladeshi Midwives Save Lives in the Rohingya Refugee Camps of Cox's Bazar.

https://asiapacific.unfpa.org/en/news/bangladeshi-midwives-save-lives-rohingya-ref ugee-camps-coxs-bazar

[10] Ministry of Health and Family Welfare (2011) Three Thousand Midwives by 2015: A Strategy to Scale up the Midwifery Workforce in Bangladesh. http://www.mohfw.gov.bd

[11] Bhuiya, I., Chowdhury, A.H. and Zahiduzzaman, K.M. (2015) First Private Sector Midwifery Education Initiative in Bangladesh: Experience from the BRAC University. Journal of Asian Midwives, 2, 14-25.

https://pdfs.semanticscholar.org/0410/e375683eb40ef3e7ee4c79b9c27470fdc30e.pdf

[12] Bogren, M., Begum, F. and Erlandsson, K. (2017) The Historical Development of the Midwifery Profession in Bangladesh. Journal of Asian Midwives, 4, 65-74.

[13] Gain, S.M. (2018) Catastrophes for a Host Country. https://www.dhakatribune.com/magazine/2018/05/24/catastrophes-for-a-host-coun try

[14] Government of Bangladesh (2017) Family Planning 2020 Commitment. http://ec2-54-210-230-186.compute-1.amazonaws.com/wp-content/uploads/2018/0 4/Govt_Bangladesh_FP2020_Commitment_2017.pdf

[15] Health Sector (2019) Rohingya Crisis in Cox's Bazar District, Bangladesh: Health Sector Bulletin.

https://www.humanitarianresponse.info/sites/www.humanitarianresponse.info/files /documents/files/health_sector_bulletin_no.9.pdf

[16] UNFPA United Nations Population Fund (2019) Bangladesh Situation Report. https://bangladesh.unfpa.org/sites/default/files/pub-pdf/ext\%20sitrep\%20final\%202 5\%20April\%202019.pdf

[17] Hope Foundation for Women and Children of Bangladesh (2019). https://www.hopeforbangladesh.org

[18] Global Health Workforce Alliance (2011) Bangladesh. https://www.who.int/workforcealliance/countries/bgd/en

[19] Schnabel, L. and Huang, C. (2019) Removing Barriers and Closing Gaps: Improving Sexual and Reproductive Health and Rights for Rohingya Refugees and Host Communities. Center for Global Development, Washington DC. https://www.cgdev.org/publication/removing-barriers-and-closing-gaps-improving -sexual-and-reproductive-health-and-rights\#ftn9

[20] Joarder, T., Rawal, L.B., Ahmed, S.M., Uddin, A. and Evans, T.G. (2018) Retaining Doctors in Rural Bangladesh: A Policy Analysis. International Journal Health Policy Management, 7, 847-858. https://doi.org/10.15171/ijhpm.2018.37

[21] Chodzaza, E. and Bultemeier, K. (2010) Service Providers' Perception of the Quality of Emergency Obstetric Care Provided and Factors Identified Which Affect the Provision of Quality Care. Malawi Medical Journal, 22, 104-111. https://doi.org/10.4314/mmj.v22i4.63946

[22] (2010) Gender Equity Is the Key to Maternal and Child Health. The Lancet, 375, 1939. https://doi.org/10.1016/S0140-6736(10)60905-1

[23] Haider, M.R., Qureshi, Z.P. and Khan, M.M. (2017) Effects of Women's Autonomy on Maternal Healthcare Utilization in Bangladesh: Evidence from a National Sur- 
vey. Sexual Reproductive Healthcare, 14, 40-47.

https://doi.org/10.1016/j.srhc.2017.09.002

[24] Anwar, I., Nababan, H.Y., Mostari, S., Rahman, A. and Khan, J.A. (2015) Trends and Inequities in Use of Maternal Health Care Services in Bangladesh, 1991-2011. PLoS ONE, 10, e0120309. https://doi.org/10.1371/journal.pone.0120309

[25] Walton, L.M. and Schbley, B. (2013) Maternal Healthcare in Bangladesh and Gender Equity: A Review Article. Online Journal of Health Ethics, 9, Article 8. https://doi.org/10.18785/ojhe.0901.08

[26] Afsana, K., Rashid, S.F., Chowdhury, A.M.R. and Theobald, S. (2007) Promoting Maternal Health: Gender Equity in Bangladesh. British Journal of Midwifery, 15, 721. https://doi.org/10.12968/bjom.2007.15.11.27474

[27] Asaduzzaman, M.S. and Ali, R.N. (2015) Gender Inequality in Bangladesh. Gender Inequality, 3, 54-64.

[28] Paul, B.K. and Rumsey, D.J. (2002) Utilization of Health Facilities and Trained Birth Attendants for Childbirth in Rural Bangladesh: An Empirical Study. Sociology Scientific Medicine, 54, 1755-1765. https://doi.org/10.1016/S0277-9536(01)00148-4

[29] Koenig, M.A., Jamil, K. and Streatfield, P. (2007) Maternal Health and Care-Seeking Behavior in Bangladesh: Findings from a National Survey. International Family Planning Perspectives, 33, 75-82. https://doi.org/10.1363/3307507

[30] Kalim, N., Anwar, I., Khan, J., Blum, L.S., Moran, A.C., Botlero, R. and Koblinsky, M. (2009) Postpartum Haemorrhage and Eclampsia: Differences in Knowledge and Care-Seeking Behaviour in Two Districts of Bangladesh. Journal Health Population Nutrition, 27, 156-169. https://doi.org/10.3329/jhpn.v27i2.3328

[31] Islam, M.A., Chowdhury, R.I. and Chakraborty, N. (2004) Factors Associated with Delivery Complications in Rural Bangladesh. European Journal of Contraception \& Reproductive Health Care, 9, 203-213. https://doi.org/10.1080/13625180400017438

[32] Begum, T., Rahman, A., Nababan, H., Hoque, D., Khan, A.F., Ali, T. and Anwar, I. (2017) Indications and Determinants of Caesarean Section Delivery: Evidence from a Population-Based Study in Matlab, Bangladesh. PLOS ONE, 12, e0188074. https://doi.org/10.1371/journal.pone.0188074

[33] Save the Children (2019) Save the Children in Bangladesh Records a 51\% Rise in "Needless" C-Sections in Two Years. https://bdnews24.com/health/2019/06/20/save-the-children-in-bangladesh-records51-rise-in-needless-c-sections-in-two-years

[34] Haider, M., Rahman, M., Moinuddin, M., Rahman, A., Ahmed, S. and Khan, M. (2018) Ever Increasing Cesarean Section and Its Economic Burden in Bangladesh. PLoS ONE, 13, e0208623. https://doi.org/10.1371/journal.pone.0208623

[35] Renfrew, M.J., McFadden, A., Bastos, M.H., Campbell, J., Channon, A.A., Cheung, N.F., et al. (2014) Midwifery and Quality Care: Findings from a New Evidence-Informed Framework for Maternal and Newborn Care. The Lancet, 384, 1129-1145. https://doi.org/10.1016/S0140-6736(14)60789-3

[36] Bogren, M.U., Wigert, H., Edgren, L. and Berg, M. (2015) Towards a Midwifery Profession in Bangladesh: A Systems Approach for a Complex World. BMC Pregnancy and Childbirth, 15, 325. https://doi.org/10.1186/s12884-015-0740-8

[37] Bogren, M., Erlandsson, K., Members of the Midwifery Faculty Master's Degree Holders in Sexual and Reproductive Health and Rights and Byrskog, U. (2018) What Prevents Midwifery Quality Care in Bangladesh? A Focus Group Enquiry with Midwifery Students. BMC Health Services Research, 18, 639. 
https://doi.org/10.1186/s12913-018-3447-5

[38] Filby, A., McConville, F. and Portela, A. (2016) What Prevents Quality Midwifery Care? A Systematic Mapping of Barriers in Low and Middle-Income Countries from the Provider Perspective. PLoS ONE, 11, e0153391.

https://doi.org/10.1371/journal.pone.0153391

[39] ACNM American College of Nurse-Midwives (2018) ACNM and ACOG Announce New Joint Statement of Practice Relations. https://www.midwife.org/ACNM-and-ACOG-annouce-new-joint-statement

[40] Bogren, M. and Erlandsson, K. (2018) Opportunities, Challenges and Strategies When Building a Midwifery Profession. Findings from a Qualitative Study in Bangladesh and Nepal. Sexual Reproductive Healthcare, 16, 45-49. https://doi.org/10.1016/j.srhc.2018.02.003

[41] Joint Commission (2019) Sentinel Event Policy and Procedures. https://www.jointcommission.org/sentinel_event_policy_and_procedures 